\title{
A Diversity of Asymmetric Nano-/ Microcolloidal Architectures Grown by ATRP from Janus Seeds
}

\author{
Chengjun Kang§ and Andrei Honciuc* \\ §SCS-DSM Award for best poster presentation in Polymer, Colloids \& Interfaces
}

\begin{abstract}
The fabrication of colloids has witnessed significant progress during the last decade, however, fabrication of anisotropic colloidal particles with complex geometries still represents a challenging task. Here, we present nano-/micro-sized colloidal architectures which 'grow' directly from nanoparticle seeds by controlled radical polymerization, resembling the growth of plants from seeds in the natural world. Specifically, we use the atom transfer radical polymerization (ATRP) technique to grow colloidal architectures from snowman-shaped Janus nanoparticle seeds (JNPS). The key to this synthetic approach is the asymmetric placement of the ATRP initiators in the bulk of one JNPS lobe. By starting the polymerization, monomers continuously add to the initiator containing the JNPS lobe, which subsequently grows into a larger colloidal structure. By controlling growth conditions mainly through the interaction strength between the monomer and JNPS, a variety of colloidal architectures result, for example, dish-, basket, cocoon-, flower-, helmet- mushroom-, dumpling and pumpkin-like geometries. Furthermore, each of these grown architectures have different surface morphologies, including smooth-, island- and grouped island nanostructures. The present work provides an alternative method to the synthesis of anisotropic particles with complex geometries and tunable surface morphologies, thus enriching the toolbox for the colloid synthesis.
\end{abstract}

Keywords: Anisotropic colloids · ATRP · Janus nanoparticles · Surface nanostructures

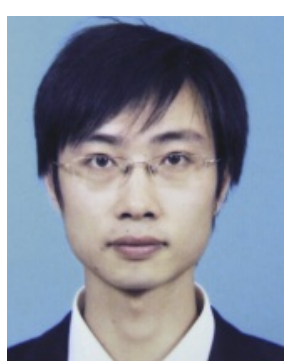

Chengjun Kang was born in Sichuan (China) in 1985. He came to Switzerland to study at ETH Zurich in 2012, under the supervision of Prof. Nicolas Spencer at the Laboratory of Surface Science and Technology, where he received his $\mathrm{PhD}$ degree in 2016. His doctoral study focused on the design, synthesis and application of photo-cleavable atom transfer radial polymerization (ATRP) initiators. From July 2016, he joined the group of Prof. Honciuc Andrei at the Zurich University of Applied Sciences. During this time, his research topic is on Janus nanoparticles (JNPs) synthesis and application, with special attention paid to the synthesis of JNPs with unique structures and self-assembly properties.

\section{Introduction}

Janus nanoparticles (JNPs) have at least two distinct chemical and/or physical properties on different parts of the same particle. ${ }^{[1]}$ Compared to conventional homogeneous nanoparticles (NPs) which have one property on their surfaces, JNPs have a much wider application potential in many interesting fields such as selfassembly and directed self-assembly, ${ }^{[2]}$ microprobes for imaging complex micro environments, ${ }^{[3]}$ nano-robotics, ${ }^{[4]}$ solid surfactants and emulsifiers, ${ }^{[5-8]}$ or as building-blocks for generating further structures such as nano-/microcolloidal architectures, the subject of the current work.

\footnotetext{
${ }^{\star}$ Correspondence: Prof. A. Honciuc E-mail: Andrei.honciuc@zhaw.ch

Institute of Chemistry and Biotechnology; Zurich University of Applied Sciences Einsiedlerstrasse $31, \mathrm{CH}-8820$ Wädenswil
}

\section{Growth of Nano-/Microcolloidal Architectures from Janus Nanoparticle Seeds}

Anisotropic character is prevalent in the natural world, anisotropic nano-/micro particles represent an important model to understand asymmetry in natural structures. ${ }^{[9-11]}$ The toolbox of methods for the synthesis of colloidal particles expanded significantly during the past decades and colloidal particles with various anisotropic geometries have been obtained: snowman, ${ }^{112,13]}$ disc-, ${ }^{[14,15]}$ crescent moon-, ${ }^{[16]}$ pistachio-, ${ }^{[16]}$ and lens-shaped Janus nanoparticles ${ }^{[17]}$ are the typical examples. However, it is still a great challenge to directly synthesize asymmetric colloidal particles with hierarchical structures.

Atom transfer radical polymerization (ATRP) was discovered in $1995,{ }^{[18,19]}$ since then ATRP methods were extensively employed for the fabrication of well-defined polymers or block copolymers. ${ }^{\text {[20] }}$ One of the most important components for a successful ATRP reaction is the initiator, which often contains halogen species, which is typically homogeneously distributed in a solvent.[21,22] The solvent dispersed initiators grow into polymer chains by the addition of monomers. The application of ATRP has been further expanded, by immobilizing the initiators on surfaces, enabling grafting of polymers on various materials. As compared to the initiators freely dispersed in solution, the freedom of surface grafted initiators is significantly reduced. Thus, polymer chains grown from these surface immobilized initiators adopt a brush-like conformation, and thus earn the name 'polymer brushes'. ${ }^{[23]}$ ATRP initiators could completely lose their freedom by burying them entirely in a solid bulk polymer; however, the growth of polymer chains from these type of 'imprisoned' initiators is much less explored. In the present work, by selectively distributing ATRP initiators in the bulk of one of the lobes of a snowman-shaped Janus, we take an alternative approach to the synthesis of anisotropic nano-/micro-sized architectures with tunable complex geometries and surface nanostructures. 


\section{Synthetic Strategy for Growing Nano-/ Microcolloidal Architectures}

To grow asymmetric micro-/nano- colloidal architectures we concentrate ATRP initiators in one lobe of a snowman-shaped Janus nanoparticle and refer to these as Janus nanoparticle seeds (JNPS) (Fig. 1a). During the polymerization process, monomers continuously and selectively add to the JNPS lobe containing the initiators. The inherent asymmetry of the JNPS promotes further directional growth into nano-/micro-sized architectures. Narrowly polydispersed JNPS with ATRP initiators concentrated in one lobe were first fabricated. Previously, we used seeded emulsion polymerization (SEP) to produce monodispersed snowman-shaped Janus nanoparticles, under surfactant-free conditions and in gram scale amount, in which the formation of a new lobe takes place by phase separation of the second type monomer from the starting seed nanoparticle (NP). ${ }^{[2,25]}$ Inspired by this, in the present study, ATRP initiators were used as the second monomer to form a new Janus lobe from seed nanoparticles; we used the mixture of (3-mercaptopropyl) trimethoxysilane (MTS) and ATRP initiator 2- bromo-2-methyl-N -(3-(trimethoxysilyl) propyl) propanamide (BMTP).[26,27] MTS was used because it leads to phase separation on different types of seed NPs surfaces. ${ }^{6,12,13]}$ Additionally, the MTS and the BMTP molecules mix well, meaning they have a proper 'bonding force' that is strong enough to form a solid Janus lobe and weak enough to allow for the expansion of the lobe due to the addition of monomers during ATRP polymerization, which is the key feature for further growth of the JNPS into nano-/microcolloidal architectures.

\subsection{Tuning the Structure of the Grown Polymeric Colloidal Architecture}

The diversity of nano-/micro-sized colloidal architectures grown by ATRP can be controlled by several parameters: $(i)$ physical morphology given by the relative size ratio of the lobes in the JNPS, (ii) the chemical compositions of the seed NPs from which JNPS are made and (iii) the ATRP initiator concentration in the initiation lobe.

First, we examined the influences of the JNPS morphologies on the colloidal architectures growth; for this, relative size between the seed NPs lobe and the MTPS/BMTP lobe was adjusted, while other parameters, e.g. the volume ratio between the MTS and the BMTP was fixed at 3:2, cross-linking degree of NPs having $90 \%$ poly (tert -butyl acrylate) (PtBA) and $10 \%$ polystyrene (PS) with identical diameters, remained the same.

The PtBA-MTS/BMTP JNPS with different MTS/BMTP lobe sizes were synthesized by adjusting the volume from $0.5 \mathrm{ml}$ to 2.5 $\mathrm{ml}$ of monomers added per $1.0 \mathrm{~g}$ of seed NPs (Fig. 1a, Fig. 2a,c). ${ }^{[27]}$ 2-Hydroxylethyl methacrylate (HEMA) was used as monomer to promote the formation of colloidal architectures, this is because HEMA monomers have a very fast ATRP polymerization rate in aqueous environment. [28-30]

For instance, from $1.0 \mathrm{~g}$ seed NPs and $0.5 \mathrm{ml}$ MTS/BMTP mixture a MTS/BMTP Janus lobe with a diameter of $159 \pm 5.1$ nm was obtained. Next, with HEMA these JNPS grow into disclike colloids with $1.3 \pm 0.1 \mu \mathrm{m}$ in diameter (Fig. 1a, Fig. 2b). The geometry of the grown colloidal resembles that of a mushroom, with the original seed NP occupying the center of the disc architecture (Fig. 2b).

By increasing the volume of the MTS/BMTP from $0.5 \mathrm{ml}$ to $2.5 \mathrm{ml}$ per $1.0 \mathrm{~g}$ seed NPs, the newly formed JNPS MTS/ BMTP lobe increases in size to $423 \pm 16.1 \mathrm{~nm}$ (Fig. 1a, Fig. 2c). The colloidal architectures grown from the bigger MTS/BMTP lobe, are also bigger, e.g. $3.9 \pm 1.2 \mu \mathrm{m}$ (Fig. $2 \mathrm{~d}$ ), and have the appearance of a basket with a smooth surface (Fig. 2d). As also observed in the disk-like colloidal architecture, each basket-like colloidal structure contains one seed NPs inside. These observa-
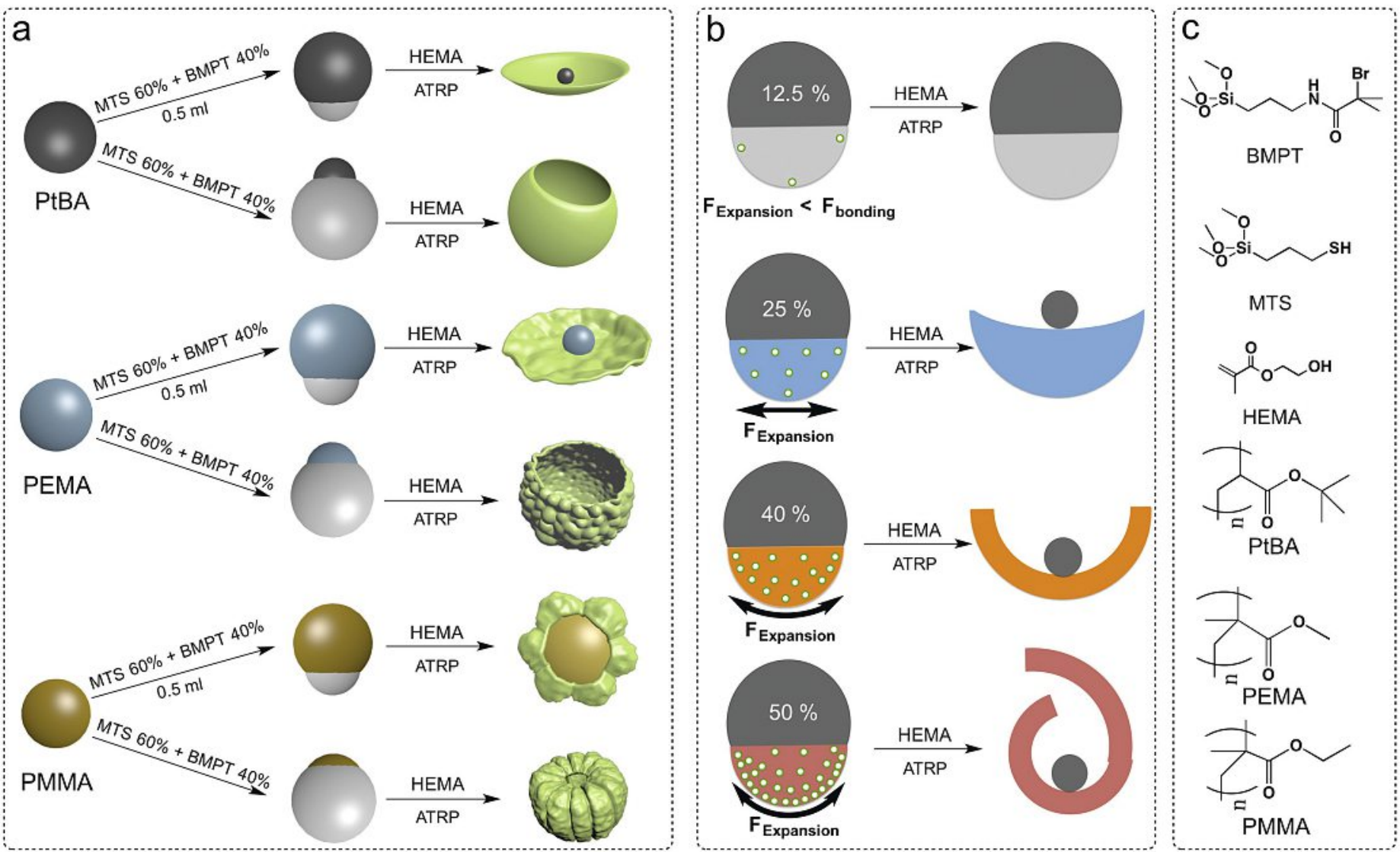

Fig. 1. (a) Growth of colloidal architectures by using different seed NPs and different MTS/BMTP lobe sizes via ATRP. (b) Influence of BMTP concentrations in the MTS/BMTP lobe on the curvature of the grown colloidal architectures. (c) Chemicals used in the present experiments. Adapted with permission from ref. [28]. Copyright 2018 American Chemical Society. 
tions demonstrate the relationship that exists between the size of the MTS/BMTP JNPS lobe and the geometry of the grown polymeric colloidal architectures. Control experiments did not show any obvious influences of reaction conditions, such as $\mathrm{pH}$ and polymerization time, on the formation of colloidal architectures.

Next, the influence of the MTS/BMTP Janus lobe compositions on the growth of colloidal architectures was examined, namely by adjusting the ratios between the MTS and BMTP monomers.

To achieve growth of JNPS into colloidal architectures, the expansion forces due to the addition and polymerization of the HEMA monomer should be larger than the 'bonding forces' between the MTS and the BMTP molecules inside the Janus initiation lobe. The expansion force of polymerization is determined by the ATRP initiator concentration in the JNPS lobe. For this, we synthesized JNPS with feeding BMTP volume concentrations of $12.5 \%, 25 \%, 40 \%$, and 50\%, respectively (Fig. 2i-1), while other parameters were fixed, e.g. PtBA-MTS/BMTP JNPS with the same PtBA seed NPs and identical MTS/BMTP lobe sizes. ${ }^{[28]}$ The JNPS containing 12.5\% BMTP in the MTS/ BMTP lobe did not grow into larger colloidal architectures (Fig. 2i). JNPS start to grow into larger colloidal architectures when the BMTP concentration reaches $25 \%$, which indicates that the growth of colloidal architectures takes place when the initiator concentration is above a critical value, such that the expansion force generated by the addition of HEMA monomers is larger than the 'bonding force' between the MTS and BMTP. Furthermore, above the critical concentration of the initiator, different colloidal architectures could
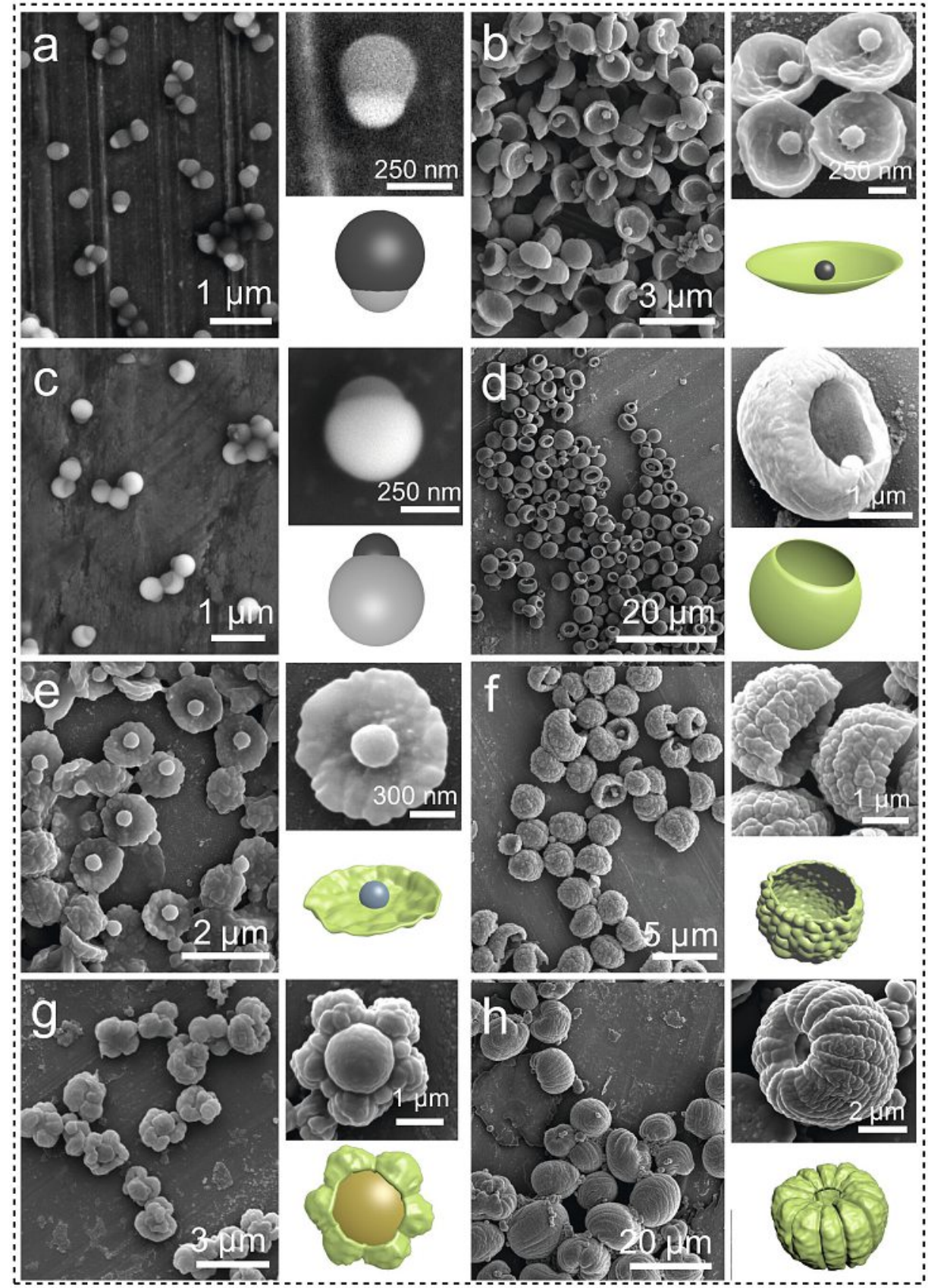
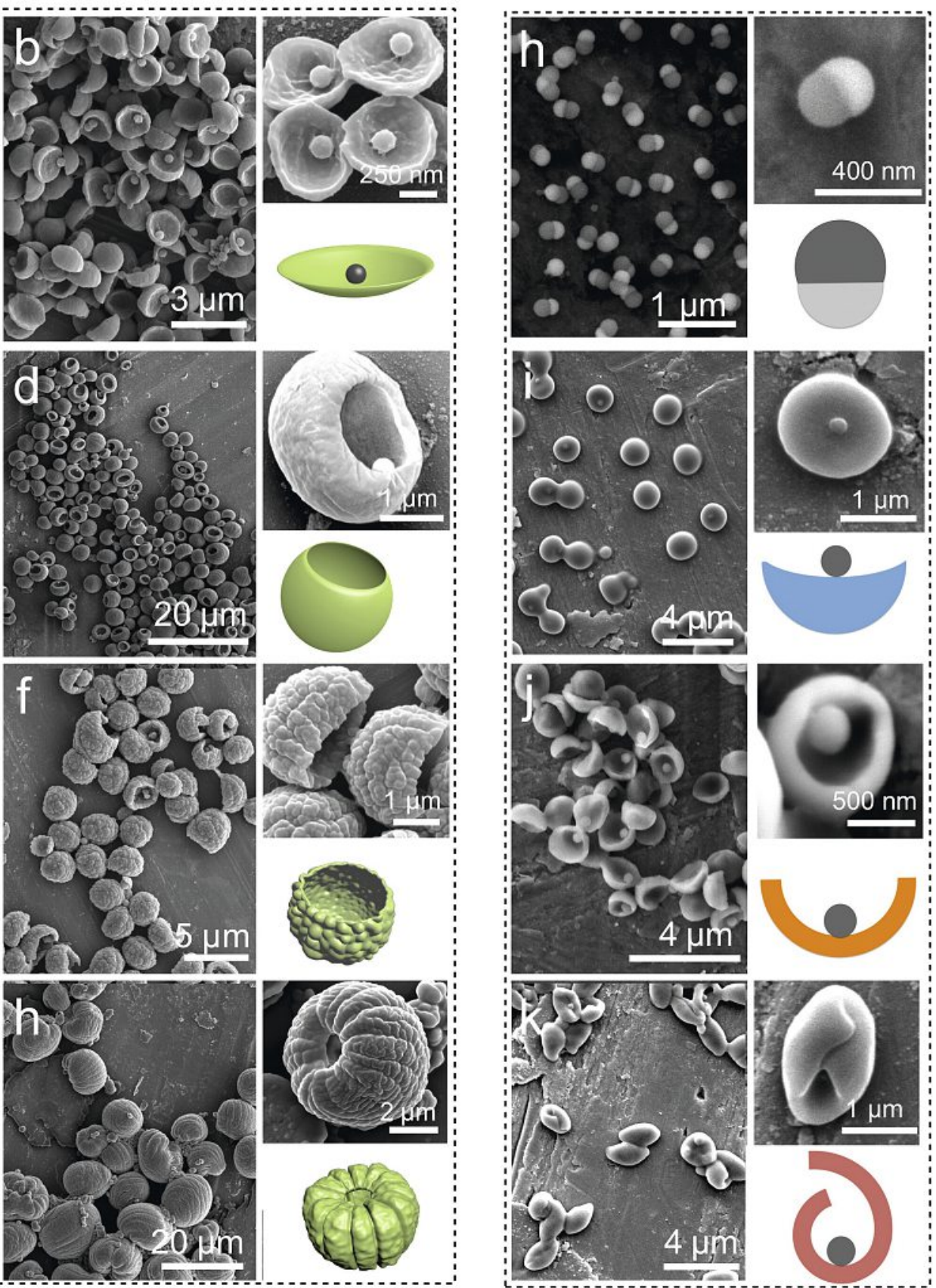

Fig. 2. SEM images of (a) PtBA-MTS/BMTP JNPS with one small MTS/BMTP lobe. (b) Disc-like colloidal architectures grown from (a). (c) PtBA-MTS/ BMTP JNPS with one big MTS/BMTP lobe. (d) Basket-like colloidal architectures grown from (c). (e) Disc-like colloidal architectures with rough surface nanostructures grown from PEMA-MTS/BMTP JNPS with small MTS/BMTP lobe size. (f) Basket-like colloidal architectures with rough surface nanostructures grown from PEMA-MTS/BMTP JNPS with big MTS/BMTP lobe size. (g) Flower-like colloidal architectures grown from PMMA-MTS/ BMTP JNPS with small MTS/BMTP lobe size. (h) Pumpkin-like colloidal architectures grown from PMMA-MTS/BMTP JNPS with big MTS/BMTP lobe size. (i-l) Colloidal structures grown from PtBA-MTS/BMTP JNPS with 15\%, 25\%, 40\% and 50\% (v/v) BMTP concentrations in the BMTP/MTS lobe. Adapted with permission from ref. [28]. Copyright 2018 American Chemical Society. 
be obtained from JNPS with different BMTP concentrations (Fig. $2 j-1)$. For example, while the solid hemispheric colloidal architectures with the seed NPs on the top of the shallow concave side can be grown from JNPS with 25\% BMTP (Fig. 2j), basketlike colloidal architectures were obtained from the JNPS with $40 \%$ BMTP (Fig. 2k), indicating the increased BMTP concentration will largely increase the curvature of the grown architectures. This is confirmed by the observation of dumpling-like architectures that were grown from the JNPS containing 50\% BMTP; these dumpling-like colloidal architectures form from highly curved and folded outer shell layers, completely wrapping the original seed NPs inside (Fig. 21).

The above experiments demonstrate that the BMTP initiator concentration in the Janus lobe has a great influence on the curvature of the ATRP grown colloidal architectures. This can be explained by the difference between the HEMA monomer diffusion speed and its consumption during the ATRP polymerization. Specifically, for a low initiator concentration (25\%), HEMA is consumed with a relatively low speed, therefore, the monomer diffusion speed is high enough to deliver sufficient amount of monomers into the every parts of the growing MTS/BMTP lobe. This results in an evenly growing MTS/BMTP lobe leading to the formation of colloidal architectures similar in geometry to the initial MTS/BMTP lobe, but much larger in diameter (Fig. 2j). As the BMTP initiator concentration increases, the HEMA monomer is consumed with a higher speed during the polymerization process, while the monomer diffusion speed remains the same. Therefore, in contrast to the initiation centers buried inside the growing colloidal architecture, HEMA monomers will reach more easily those points that are closer to the growing MTS/BMTP lobe surface (Fig. 1b, Fig. 2k). This will lead to the difference in the growth rate between the outer and the deeper layer of the colloidal architectures; ultimately the difference in the expansion rate will force the colloidal architectures to bend. This is the reason why for a BMTP concentration of 50\%, the outer layers of the colloidal architecture bend until they overlap with each other and wrap the seed NPs inside (Fig. 1b, Fig. 2l).

Next, we investigated the influence of the seed NPs surface chemical composition on the growth of colloidal architectures. It is well known that the phase separation of monomers from seed resulting in the formation of snowman-shaped JNPs by SEP is directly influenced by the surface chemistry of original seed NPs. In the previous experiments, we have shown that the JNPS containing the PtBA seed NPs lobes and the MTS/BMTP initiation lobes have complete phase separation, indicating relatively weak attraction forces between these two lobes. After the polymerization of the PtBA-MTS/BMTP JNPS, colloidal architectures with smooth surfaces were obtained. Alternately, we hypothesized that if the phase separation between the seed NP and the MTS/BMTP lobe is incomplete, the attraction forces between the two lobes will be stronger and larger expanding forces will be needed for the growing MTS/BMTP lobe. This counteracting forces between the expansion of the initiation lobe and attraction to the second lobe might lead to interesting colloidal architectures. To show this, we used poly(ethyl methacrylate) (PEMA) as seed NPs, which is more hydrophilic than the PtBA NPs; the increased hydrophilicity results in less phase separation between the PEMA lobe and the MTS/BMTP lobe, therefore stronger interactions between these two lobes can be expected. Similar to the PtBA-MTS/BMTP JNPS, the PEMA-MTS/BMTP JNPS of various MTS/BMTP lobe sizes can be obtained simply by tuning the ratios between the feeding amounts of the PEMA seed NPs and feeding volume of the MTS/BMTP monomer. ${ }^{[28]}$ Based on the previous experience of the PtBA-MTS/BMTP JNPS, it is not surprising that the PEMAMTS/BMTP JNPS with small and big MTS/BMTP lobes can also grow into colloidal architectures having disc- and basket-like structures, respectively. However, these colloidal architectures showed rather rough surfaces with island-like nanostructures (Fig. 1a, Fig. 2e,f), in sharp contrast to the smooth surfaces of the colloidal architectures grown by the PtBA-MTS/BMTP JNPS (Fig. 1a, Fig. 2b,d). The formation of the rough surface structures is very likely due to the strong attraction forces that exist between the growing MTS/BMTP lobe and the PEMA seed NP surface. When the phase separation between the seed NPs and the MTS/ BMTP lobe is complete and the attraction strength between the two lobes is weak, e.g. PtBA-MTS/BMTP JNPS, the minimum expanding forces required for the detachment of the growing MTS/ BMTP lobe from seed NPs result in colloidal architectures with smooth surfaces. However, as the attraction forces between the two lobes increase, e.g. PEMA-MTS/BMTP, the enhanced attraction force restrict the expansion of the growing MTS/BMTP lobe; as a result, the internal stress distort the surface morphologies of the grown colloidal architectures. The above assumption is verified by the growth of JNPS with even more polar seed NPs, namely poly(methyl methacrylate) (PMMA) seed NPs. Because of increased polarity, the phase separation between the PMMA NPs and the MTS/BMTP lobe is the least among JNPS used in the present study, indicating the strongest attraction strength between the PMMA lobe and MTS/BMTP lobe. In fact, when the PMMAMTS/BMTP JNPS with small MTS/BMTP lobes were used, the expansion force generated by the growing MTS/BMTP lobe is not as strong as the attraction force, therefore, the growing MTS/ BMTP lobe cannot detach from the PMMA NPs surface. The strong inner stress split the growing MTS/BMTP lobe into several parts, forming flower-like geometry (Fig. 1a, Fig. 2g). The JNPS with larger MTS/BMTP lobe size could make the colloidal structures grow much bigger and significantly increase the expansion force. Pumpkin-like colloidal architectures were obtained from the growth of PMMA-MTS/BMTP JNPS containing larger MTS/BMTP lobe sizes (Fig. 2h). These pumpkin-like structures have island-like surface nanostructures as has been observed in the previous cases, the difference is that the present island structures are divided into several groups by concave lines running from one pole of the colloidal architecture to the other.

\subsection{Potential Applications}

The current method shows great potential for many interesting applications, for example, colloids with rather complex structures can be synthesized, which are difficult to fabricate by alternative methods. Specifically, complex structures can be fabricated by careful design of the starting JNPS. To illustrate this, JNPS containing two identical seed NPs can be synthesized with a yield of $20 \%$ (Fig. 3a,b ). [31] The growth of this special type of JNPS results in colloidal architectures with helmet-like morphology, in which each seed NP results in a cavity structure (Fig. 3c,d). Furthermore, another type of JNPS containing two MTS/BMTP initiation lobes attaching to one type of seed NPs can be fabricated; ${ }^{[32]}$ each of these two initiation lobes can grow into a colloidal architecture independently. The overall obtained colloidal structures depend on the relative size of these two MTS/BMTP initiation lobes. Specifically, when these two MTS/BMTP lobes have similar sizes (Fig. 3a,h), this type of JNPS can grow into colloidal architectures with cocoon-like geometries (Fig. 3a,i,j), in which the two grown basket-like architectures of similar sizes have their openings in close contact with each other. In another case, JNPS having one MTS/BMTP lobe obviously larger than the other (Fig. 3a,e) can grow into hierarchical structures with one disc-like colloidal structure contained in the other larger basketlike colloidal structure. In this case the disc-like structure grows from the smaller MTS/BMTP lobe, while the basket-like structure grows from the larger MTS/BMTP lobe (Fig. 3a,f). Another interesting application of the current technique is that the synthesized basket-like colloidal architectures showed interesting selfassembly properties (Fig. $3 \mathrm{k}-\mathrm{n}$ ). We observed that the randomly 

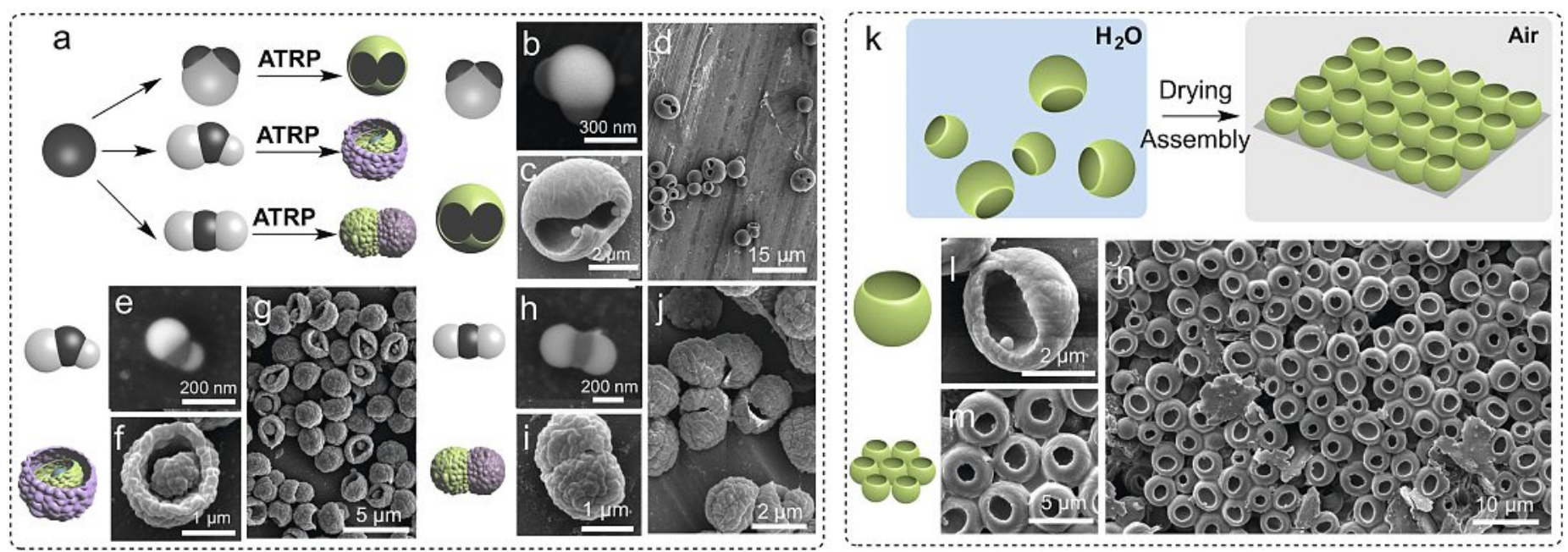

Fig. 3. (a) Scheme showing the synthesis of hierarchical colloidal architectures. (b) JNPS containing two seed NPs. (c, d) Helmet-like colloids produced from (b). (e) JNPS containing one seed NPs and two ATRP initiator loaded Janus lobes with different sizes. (f, g) Colloids produced from (e), which are formed by two basket-like colloids with their openings in close contact with each other. (h) JNPs containing one seed NPs and two ATRP initiator loaded Janus lobes with same size. (i, j) Colloids produced from (h), which consist of one basket-like colloid architecture containing a disc-like colloid with a spherical seed NP inside. (k) Basket-like colloid containing one spherical NP inside. (l, m) Basket-like colloid assembly upon drying. The different colors in the cartoon image intend to show colloidal architectures that are grown from different initiation Janus lobes. These colloidal architectures have the same chemical compositions. Adapted with permission from ref. [28]. Copyright 2018 American Chemical Society.

dispersed basket-like colloidal architectures in aqueous solution would automatically assemble upon drying, their arrangement is in such a manner that the 'baskets' hexagonally packed together with over $90 \%$ orientated by having their openings toward air. Further study to elucidate why these 'baskets' adopt an oriented configuration is in progress.

\section{Conclusion}

In summary, we have demonstrated the synthesis of asymmetric nano-/micro colloidal architectures with the ATRP technique from Janus nanoparticles as initiators. Previously, ATRP has mainly been employed for the synthesis of well-defined polymer chains both in solution and on surface of materials; the present study expands the ATRP application scope by incorporating ATRP initiator into the solid bulk of a Janus particle. The present study also provides an alternative way for the fabrication of colloids, one distinct advantage this method has is its controllability over the surface nanostructures of the synthesized colloids, in sharp contrast to the conventional colloidal synthetic methods by which only colloids with smooth surfaces can be fabricated. Furthermore, the current method is interesting also because it provides the possibility to fabricate a series of asymmetric colloidal architectures, including hierarchical ones. It is thus safe to say that this work provides a different path to generate various asymmetric colloidal particles with unusual geometries and functionalities in a controlled manner.

Received: January 30, 2019

[1] A. Walther, A. H. E. Müller, Chem. Rev. 2013, 113, 5194, DOI: 10.1021/ cr300089t.

[2] Q. Chen, S. C. Bae, S. Granick, Nature 2011, 469, 381, DOI: 10.1038/nature09713.

[3] K. V. Edmond, M. T. Elsesser, G. L. Hunter, D. J. Pine, E. R. Weeks, Proc. Natl. Acad. Sci. 2012, 109, 17891, DOI: 10.1073/pnas.1203328109.

[4] S. Lach, S. M. Yoon, B. A. Grzybowski, Chem. Soc. Rev. 2016, 45, 4766, DOI: $10.1039 / \mathrm{C} 6 \mathrm{CS} 00242 \mathrm{~K}$.

[5] L. C. Bradley, K. J. Stebe, D. Lee, J. Am. Chem. Soc. 2016, 138, 11437, DOI: 10.1021/jacs.6b05633

[6] D. Wu, A. Honciuc, ACS Appl. Nano Mater. 2018, 1, 471, DOI: 10.1021/ acsanm.7b00356.

[7] D. Wu, J. W. Chew, A. Honciuc, Langmuir 2016, 32, 6376.

[8] D. Wu, B. P. Binks, A. Honciuc, Langmuir 2018, 34, 1225, DOI: 10.1021/ acs.langmuir.7b02331.
[9] Y. J. Lee, H. Yi, W.-J. Kim, K. Kang, D. S. Yun, M. S. Strano, G. Ceder, A. M. Belcher, Science 2009, 324, 1051, DOI: 10.1126/science.1171541.

[10] J. A. Lukin, G. Kontaxis, V. Simplaceanu, Y. Yuan, A. Bax, C. Ho, Proc. Natl. Acad. Sci. 2003, 100, 517, DOI: 10.1073/pnas.232715799.

[11] A. Tromans, Nature 2001, 411, 33, DOI: 10.1038/35075187.

[12] C. Kang, A. Honciuc, ACS Nano 2018, 12, 3741, DOI: 10.1021/ acsnano.8b00960.

[13] C. Kang, A. Honciuc, J. Phys. Chem. Lett. 2018, 9, 1415, DOI: 10.1021/acs. jpclett.8b00206.

[14] S. Sacanna, M. Korpics, K. Rodriguez, L. Colón-Meléndez, S.-H. Kim, D. J. Pine, G.-R. Yi, Nat. Commun. 2013, 4, ncomms2694, DOI: 10.1038/ncomms2694.

[15] M. Marechal, R. J. Kortschot, A. F. Demirörs, A. Imhof, M. Dijkstra, Nano Lett. 2010, 10, 1907, DOI: 10.1021/n1100783g.

[16] J.-B. Fan, Y. Song, H. Liu, Z. Lu, F. Zhang, H. Liu, J. Meng, L. Gu, S. Wang, L. Jiang, Sci. Adv. 2017, 3, e1603203, DOI: 10.1126/sciadv.1603203.

[17] W.-H. Chen, F. Tu, L. C. Bradley, D. Lee, Chem. Mater. 2017, 29, 2685, DOI: 10.1021/acs.chemmater.7b00494.

[18] M. Kato, M. Kamigaito, M. Sawamoto, T. Higashimura, Macromolecules 1995, 28, 1721, DOI: 10.1021/ma00109a056.

[19] J.-S. Wang, K. Matyjaszewski, J. Am. Chem. Soc. 1995, 117, 5614, DOI: $10.1021 / \mathrm{ja} 00125 \mathrm{a} 035$

[20] K. Matyjaszewski, J. Xia, Chem. Rev. 2001, 101, 2921, DOI: 10.1021/ cr940534g.

[21] K. Matyjaszewski, Macromolecules 2012, 45, 4015, DOI: 10.1021/ ma3001719.

[22] C. Kang, L. Yu, G. Cai, L. Wang, H. Jiang, J. Polym. Sci. Part Polym. Chem. 2011, 49, 3595, DOI: 10.1002/pola.24798.

[23] M. Krishnamoorthy, S. Hakobyan, M. Ramstedt, J. E. Gautrot, Chem. Rev 2014, 114, 10976, DOI: $10.1021 / \mathrm{cr} 500252 \mathrm{u}$.

[24] V. Mihali, A. Honciuc, Adv. Mater. Interfaces 2017, 4, 1700914, DOI: 10.1002/admi.201700914.

[25] W. Smulders, M. J. Monteiro, Macromolecules 2004, 37, 4474, DOI: $10.1021 / \mathrm{ma} 0494961$.

[26] G. Panzarasa, G. Soliveri, K. Sparnacci, S. Ardizzone, Chem. Commun. 2015, 51, 7313, DOI: $10.1039 / \mathrm{C} 5 \mathrm{CC} 00255 \mathrm{~A}$.

[27] C. Huang, K. Gee Neoh, E.-T. Kang, B. Shuter, J. Mater. Chem. 2011, 21, 16094, DOI: $10.1039 / \mathrm{C} 1 \mathrm{JM} 11270 \mathrm{H}$.

[28] C. Kang, A. Honciuc, Chem. Mater. 2018, 30, 7664, DOI: 10.1021/acs. chemmater.8b02946.

[29] K. L. Robinson, M. A. Khan, M. V. de Paz Báñez, X. S. Wang, S. P. Armes, Macromolecules 2001, 34, 3155, DOI: 10.1021/ma0019611

[30] C. Kang, S. N. Ramakrishna, A. Nelson, C. V. M. Cremmel, H. vom Stein, N. D. Spencer, L. Isa, E. M. Benetti, Nanoscale 2015, 7, 13017, DOI: 10.1039/ C5NR03147H.

[31] D. J. Kraft, W. S. Vlug, C. M. van Kats, A. van Blaaderen, A. Imhof, W. K. Kegel, J. Am. Chem. Soc. 2009, 131, 1182, DOI: 10.1021/ja8079803.

[32] J.-G. Park, J. D. Forster, E. R. Dufresne, Langmuir 2009, 25, 8903, DOI: $10.1021 / 1 \mathrm{la} 901969 \mathrm{~d}$. 